\title{
Current Scenario of Herbal Industry in Malaysia
}

\author{
Harisun Ya'akob ${ }^{1,2}$, Jin Ru Lim ${ }^{1}$ and Nur Athirah Hashim ${ }^{2}$ \\ ${ }^{1}$ Department of Bioprocess Engineering, Faculty of Chemical and Energy Engineering, Universiti \\ Teknologi Malaysia, 81310, Skudai, Johor, Malaysia. \\ ${ }^{2}$ Institute of Bioproduct Development, Faculty of Chemical and Energy Engineering, Universiti \\ Teknologi Malaysia, 81310, Skudai, Johor, Malaysia.
}

\begin{abstract}
The changing trend towards the use of natural ingredients in product formulation has becoming very significant nowadays. Herbal products have gained increasing popularity in the last decade, and are now used by approximately $20 \%$ of the population and contribute to the health care of our people. The natural products global trade is projected to be over RM2 trillion by the year 2020 as more people are turning to herbal-based products as nutritional and dietary supplements; an alternative to the conventional therapeutic medicine. Therefore it is hoped that the utilisation of natural ingredients could be capitalised for the mass production of herbal based products. Malaysian herbal product market is experiencing tremendous growth. Malaysia's rich tropical biodiversity is a reliable source for natural health products, and the Government is now addressing its full potential. Although herbs are often perceived as "natural" and therefore safe, many safety issues and side effects have been reported owing to active ingredients, contaminants, or interactions with modern drugs. Before herbal-based products are commercialized, several processes/steps which are critical aspects of the development and marketing of product must be addressed. Thus, there is an urgent need to conduct experimental research to provide scientific evidence in terms of safety, efficacy and quality of herbal medicines. In addition the plants as sources for new lead structure for drug development especially the active compounds that are beneficial for human health requires further investigation.
\end{abstract}

\section{Key words: Natural ingredient, herbal based product, safety, efficacy, quality}

\section{INTRODUCTION}

Since the beginning of human civilization, herbs have been an integral part of society, valued for both their culinary and medicinal properties. In recent years, interest in traditional medicine has increased greatly among researchers and the general public [1]. The considerable interest for replacing synthetic drugs with natural sources from parts of plants has led to intensified exploration and research for variety of purposes to cure illness. Thousands of plants have been used traditionally to treat various diseases, thus, natural remedies have become popular, especially in the part of lower risk of adverse reaction. [2].

With the advancement in pharmaceutical research and accumulated scientific data, and past discovery of potent drug from plants, the role of herbs in medication is expected to grow steadily alongside biologics and synthetics drugs. Furthermore, two modes of herbal based products, namely herbal supplements and therapeutic herbal drugs, will always be the preferred choice cost wise [3].Compared with conventional drug, herbal medicinal products present a number of unique problems when quality aspects are considered as herbal ingredients contain a complex mixture of constituents and concentration of plant constituents can be vary depending on environmental factors and genetic factors [4].

Control of the starting materials is essential in order to ensure reproducible quality of herbal medicinal products. Herbal ingredients must be accurately identified macroscopically and microscopically comparison with authentic herbs materials. Even when correctly authenticated, it is also important to realise that different batches of same herbal ingredient may differ in quality due to a number of factors such as inter- or intraspecies variation (vary in constituents which genetically controlled and might relate to country origin), 
environmental factors (climate, growing condition, altitude, soil), time of harvesting, plant part used (different part of same plant might contain different concentration of active compound), post-harvesting factors (storage, cleaning, processing treatment), adulteration and substitution [5].

\section{ENSURING HERBS QUALITY}

In order to ensure the quality of herbal medicinal product and in addition to microscopical examination, identity test is essential. Generally, thin-layer chromatography is used for identification purposes and it is valuable for the qualitative determination of small amounts of impurities [6]. As thin-layer chromatography is effective, easy to perform and equipment required is inexpensive, hence, this technique is served as simple chemical test and is frequently used for evaluating herbal materials and herbal preparations. Meanwhile, for identification of herbal ingredients which contain volatile compound, gas-liquid chromatography might be used and chromatogram obtained from tests might be used as fingerprint of herbal ingredient. Furthermore, the herbal ingredients with known active principles, an assay should be established to set a criterion for the acceptable minimum concentration of bioactive compounds. Gas-liquid chromatography and high performance liquid chromatography are the methods used to establish the assay of herbal ingredients [7].

In addition, herbal ingredients should be free from visible signs of contamination by moulds or insects, other animal contamination and excreta. It is seldom possible to obtain marketed plant materials that are totally free from contaminant, however, no poisonous, no dangerous or harmful foreign matter might be allowed [6]. Macroscopic examination can be employed for determining the presence of foreign matter or residues in herbal plant material while microscopy is necessary for determining the presence of powdered residue.

The presences of sand that adhering to plant surface can be identified by determine the acid-insoluble ash value of herbal materials. Acid-insoluble ash is the residue obtained after the total ash of herbal materials is treated with dilute hydrochloric acid and this measure the amount of silica present, especially as sand [8]. Other contaminants such as foreign organic matter, microbial contamination, pesticides and toxic metal should be examined through batch and batch of herbal materials. Standard and procedure should be set in order to limit the percentage of foreign organic matter contaminant such as unwanted parts of plant or other plant. Normally, aerobic bacteria and fungi are present in plant material and might increase due to improper growing practice, harvesting and collecting practice, storage condition or processing. Aerobic bacteria and fungi (yeasts and moulds) are determined by the total viable count (TVC) which represent the quantitative data of microorganism. The methods used to study TVC of microorganism include membrane-filtration, plate count and serial dilution. Microorganism such as Salmonella, Pseudomonas aeruginosa, Staphylococcus aureus and Clostridium have been identified and shown to contaminate herbal material [9], hence, it is necessary that maximum permitted level of microbial contamination is set for herbal material.

Herbal material that grown as cultivated crop might be contaminated by pesticides. Gas chromatography (GC) and coupled with mass spectrometry (MS) are methods used determined pesticides residues. Moreover, most of the pesticides contain chlorine or phosphorus in the molecule that can be detected by the measurement of total organic chloride and analysis of total organic phosphorus respectively. Toxic metals such as lead, mercury and arsenic have been found and contaminate herbal material. Lead is naturally found in earth crust and related to public health problem [10]. Mercury is found in air, water and soil, it has been studied that mercury has a toxic effect on nervous, digestion and immune system [11]. Arsenic is widely distributed in the groundwater and exposure to arsenic might to lead to cancer and skin lesions [12]. Hence, limit tests for toxic metal detection is essential for herbal material.

In a nut shell, quality of herbal products can be ensured by control the quality of herbal materials and by adherence to good manufacturing practices standards. In addition, stability test and shelf-life of products should be examined in order to determine the quality of product.

\section{STANDARDIZATION OF HERBAL PROCESSING}

Standardization of herbal medicines is the process of prescribing a set of standards, constant parameters, definitive qualitative and quantitative values that carry an assurance of quality, efficacy and safety. Standardization of herbal process involved in the physicochemical evaluation of crude drug covering aspects, such as selection and handling of raw material, safety, efficacy and stability assessment of finished product, documentation of safety and risk [13]. In manufacture of herbal product, there are four elements that needed to standardise which include raw material, plantation, processing and product development.

Generally, drugs and medicines have being a sensitive substances regarding to public health issues which strictly regulated by national regulatory. It is necessary 
to ensure that source of medicinal plant material is identified and medicinal plant raw material part which used for herbal medicine manufacturing must be obtained from the suppliers that have been followed national protocol and approved by the government authorities. Nowadays, increasing in complexity and global supply chain might bring the threat of raw material fraud which may affect the quality of final product and potentially harmful to public health [14]. Fraud of raw materials means the intentionally and economically-motivated adulteration of raw materials that involve removal or replacement of authentic substances or fraudulent addition of non-authentic substances for economic gain of the seller. Basically, there are two main type of economically motivated adulteration are sale of raw material which is unfit and potentially harmful and deliberate mislabelling of material. Furthermore, storage condition also plays an important role in establishing and improving the quality, safety and efficacy of medicinal plant material. There shall be have an adequate space for different type of material in storehouse, 'approved' and 'rejected' medicinal plant material should be stored in arrangements, temperature and humidity of store house should be controlled, proper ventilation and free from dampness [15].

Plantation of medicinal plant should followed guidelines on good agricultural practices (GAP) for medicinal plants which established by World Health Organization (WHO). The quality of medicinal plant is determined by the conditions during growth while GAP guidelines are intended to provide general technical guidance on obtaining medicinal plant materials of good quality for the sustainable production of herbal products classified as medicines. GAP include identification of cultivated medicinal plant, seed selection, issues regarding cultivation, harvesting method and personnel performance which might greatly affect the quality, safety and efficacy of medicinal plant. In addition, all organisms depend on forests for survival, besides providing habitats for animals and livelihood for humans, forest also offered watershed protection, prevent soil erosion and mitigate climate change [16].

The manufacturing process is one of the key steps where quality control is required to ensure quality of herbal medicines where good manufacturing practices (GMP) is one of the most important tools for this measure. GMP is a part of quality assurance that ensures products are consistently produced and controlled to the quality standards appropriate to their intended use and as required by marketing and national authorization and GMP is established aim at eliminating the risk inherent in medicine production. According to GMP established by WHO, all manufacturing process should clearly defined and reviewed systematically, perform qualification of critical equipment and process validation, all instructions and procedure should be written clearly with unambiguous language and critical process steps should be specified, all procedures should be carried out by trained operators, records regarding manufacture and distribution are made, products should be store at proper condition to minimize the risk that might affect the quality, a recall system of product from sale and supply should establish and complaints and reasons regarding defective products should examined. Furthermore, sanitation and hygiene also play an important role in herbal medicine process. Medicinal plant may contain microbiological contaminants due to their origin. To avoid and reduce contamination, a high level of sanitation ad hygiene during manufacture is necessary [17].

Standardization of herbal medicines formulation requires implementation of GMP [18]. Study of various parameters such as pharmacodynamics, pharmacokinetics, dosage, stability, self-life, toxicity evaluation, chemical profiling of the herbal formulations is considered essential [19]. In addition, standardization is an important aspect for maintaining and assessing the quality and safety of the polyherbal formulation as that is combination of more than one herb to attain the desire therapeutic effect. The bioactive extract of medicinal plant material that used for herbal medicines should be standardized on the basis of active principle along with chromatographic fingerprint which can be determined by high performance thin layer chromatography (HPTLC), high performance liquid chromatography (HPLC) or gas chromatography (GC) and stabilization of bioactive extract with a minimum shelf life over a year [20]. According to WHO, standardization of herbal formulations include quality control of crude drugs material, plant preparations and finished products which include three aspects: identity, purity and assay, stability assessment and shelf life, safety assessment based on experience or toxicological studies, assessment of efficacy and biological activity evaluation [19].

\section{QUALITY MEASUREMENTS FOR HERBS ACCORDING TO ISO STANDARDS}

International Organization for Standardization (ISO) is a worldwide federation of national standard bodies. ISO standard is a voluntary, non-government and market driven standard. The standard is designed to be met by organization or manufacturer that need to demonstrate the ability to consistently provide product or service that meets both customer and applicable statutory and regulatory legal requirements, aims to enhance customer satisfaction effectively and improve 
quality management system continuously and also for organization that plans to provide sustained assurance of conformity to customer and applicable requirements $[21,22]$. Whereas ISO standard pays more attention to the management of the firm compare to GMP that more focus on the manufacturing systems and validation of those systems.

On $1^{\text {st }}$ September 2005, the International Standards Organization published a single standard to encompass all the needs and designed as ISO 22000 which ensure a safe food supply chain worldwide and herbs is considered as food consumed by public [23]. Within the scope of ISO 22000, it include the organizations related to the food chain range from food manufacturers, transport, storage operator, retail and service outlets and also related to product quality and safety [24]. Therefore, quality assurance system, obligatory systems based on quality and safety of food required by law is essential to establish. Obligatory systems include Good Hygiene Practices (GHP), Good Manufacturing Practices (GMP) and Hazard Analysis and Critical Control Point (HACCP).

Good Hygiene Practices (GHP) constitute a set of guideline specifying activities to be undertaken and monitored at all steps of the food chain to ensure that the hygiene conditions is fulfilled which related to safety and quality of product. Similarly, Good Manufacturing Practices (GMP) indicates specific activity guideline in food manufacturing in order to ensure standards of food safety have been met. Hazard Analysis and Critical Control Point (HACCP) is a systematic food assurance method to identify and control food hazards. There are seven principles of HACCP which include conduct a hazard analysis, determine the critical control points (CCPs), establish critical limits, establish monitoring procedures, establish corrective actions, establish verification procedures and establish record keeping and documentation procedures [25].

In addition of obligatory systems that ensure the quality and safety, implementation of quality management system, ISO standard for quality of herbs also include transportation, storage condition, retail and service outlets and together with equipment, packaging material, labelling, cleaning agents, additive and ingredients [23]. In transportation, conveyances used for transporting herbs should be cleaned between loads. Bulk transport such as rail cars or ships should be cleaned and well ventilated to remove moisture from herbs. For storage condition, fresh herbs should be stored at appropriate low temperatures at $2-8{ }^{\circ} \mathrm{C}$; frozen products should be stored less than $-20{ }^{\circ} \mathrm{C}$ and potentially poisonous herbs should be stored separately. Processed herbs should be packaged as soon as possible to prevent deterioration and against pest attack [26].

In addition, materials used for packaging should be nonpolluting, clean, dry and in undamaged condition. Labelling of product should be clearly indicate the scientific name of herbs, the plant part, origin place, time of cultivation and collection, quantitative information and name of grower. All equipment and utensils that used in the handling of herbs should be made of materials that do not transmit toxic substances, odour and taste, non-absorbent, resistant to corrosion and capable to clean and disinfection [27]. Moreover, incorporation of additive and ingredients in products should followed national regulatory and should be obtained from supplier that has been certified and authorised.

\section{HERBAL SAFETY, QUALITY AND EFFICACY ON COMMERCIALIZATION}

In order to ensure public health, medicinal products must be safe, efficacious and of suitable quality for use. To obtain authorisation of a medicinal product within a national regulation, manufacturers of herbal medicinal product are required to demonstrate that the product produced have meet acceptable safety, quality and efficacy.

\section{Safety}

The use of herbal medicinal products might present a potential risk to human health. There are concerns that the consumer of herbal medicinal products might be exposed to potentially toxic substances either from herbal ingredients itself or as a result of exposure to contaminants present within herbal medicinal product. The safety of herbal medicinal products is important as the majority of these products are self-prescribed and is used to treat minor which generally in chronic condition. The chronic form of toxicity such as carcinogenicity, mutagenicity and hepatotoxicity are types of toxicity of most concern regarding to safety of herbal medicinal product. In addition, intrinsically toxic constituents might be present within herbal [28]. When cyanogenetic 
glycosides is being consumed and hydrolysed within gastric, hydrogen cyanide $(\mathrm{HCN})$ will be released. Hydrogen cyanide will be absorbed by gastrointestinal tract and may lead to respiratory failure. Furthermore, some herbal ingredients may cause adverse effects such as allergic, cardiac, irritant, hormonal and hepatic effect.

The potential for interaction between herbal and conventional medicines has been recognised which is one of the issues regarding to the safety of herbal medicinal product. Herb-drug interactions may be pharmacodynamics or pharmacokinetic. Pharmacodynamic interaction might occur when herbal medicine and conventional drug have similar pharmacological effects while pharmacokinetic interaction could result when a herbs alters the absorption, distribution, metabolism of drug. In addition, there is also the potential issued of herb-herb interactions where combinations of herbal ingredients are used. For example of herb-drug interaction, some reports show that interaction between St John's wort and certain medicines such as indinavir, warfarin and digoxin lead to a loss or reduction in therapeutic effect. It also has been reported that several herbal ingredients such as garlic, ginkgo and ginseng potentially interfere with warfarin. Hence, it is necessary to collect and analyse the information regarding to the safety risks associated with the use of herbal products in order to protect public health [29].

\section{Quality}

The majority of quality-related problems are associated with unregulated herbal products. There are studies show that many ethnic medicines lack effective quality controls and may give rise to serious public health concerns. The problem associated deliberate or accidental inclusion of prohibited ingredients, substitution of ingredients, contamination with toxic substance and difference between labelled and actual contents. For example in substitution of ingredients, it has been found that exposure of Aristolochia species in unlicensed herbal medicines has resulted in cases of nephrotoxicity and carcinogenicity. The toxicity is traced to the inadvertent use of the toxic Aristolochia fangchi root in the formulation as a substitute for Stephania tetranda. Aristolochia species contain a toxic component, aristolochic acids which are known to be nephrotoxic, carcinogenic and mutagenic [30-31].
In addition, adulteration of herbal medicines with heavy metals or toxic elements and synthetic drugs continues to be a major problem. In most of the cases that involving synthetic drugs, the drugs are undeclared in the medicinal products and only exposed when consumers experience adverse effect which are sufficiently serious. The heavy metals or toxic elements may arise from plant ingredients themselves or be introduced as trace contaminants during processing and also added intentionally that declared as ingredients. There are many evidences show that adulterated product is found and reported. For examples, a survey contacted between 1990 and 1997 in Singapore on Traditional Chinese Medicine (TCM) reported that 42 different products were found contain excessive amount of heavy metals such as mercury, lead and arsenic and 32 different TCM products were found to contain 19 drugs [29].

\section{Efficacy}

Biological response or efficacy is determined by concentration of active ingredients. Additional of alter component may alter or modify the activity of bioactive compound such as bioavailability, metabolism, physical properties and synergistic effects. Herbal medicinal product is often used in minor condition which related to public health. Despite there is growing consumption of herbal medicine world-wide, but there is a dearth of scientific evidence of efficacy for most herbal medicines. One of the problems of herbal medicinal product is that herbal ingredients contain a vast vary of chemical consistuents. Furthermore, herbal medicines traditionally involve mixtures of different herbal ingredients that it has been claimed that combination of herbal ingredients provides a better therapeutic outcome while reducing adverse effect and toxicity. Hence, herbal medicines intended for use in more serious medical conditions require evidence of efficacy to support their use [29].

\section{CONCLUSION}

Interest in traditional herbs for medicine used has increased substantially in Malaysia. Thus, medicinal herbs markets have been growing rapidly, and significant economic gains are being comprehended. Despite their widespread use, numerous reports show that the herbal products available to consumers are of 
variable quality. Thus, there is an urgent need to conduct experimental research to provide scientific evidence in terms of safety, efficacy and quality of herbal medicines that are beneficial for human health.

\section{REFERENCES}

[1] Fakim, A.G. Medicinal plants: Traditions of yesterday and drugs of tomorrow. 2016. Molecular Aspects of Medicine 27, 1-93.

[2] Natarajan, K.S., Narasimhan, M., Shanmugasunduraram, K.R. and Shanmugusundaram, E.R.B. 2006. Antioxidant activity of a salt-spice-herbal mixture against free radical induction. Journal of Ethnopharmacology 105, 76-83.

[3] Ahmad, F., Zaidi, M.A.S., Sulaiman, N. and Majid, F.A.A. 2015. Issues and Challenges in the Development of the Herbal Industry in Malaysia. Persidangan Kebangsaan Ekonomi Malaysia ke10 (PERKEM 10), 227-238.

[4] Shinde, V.M., Dhalwal, K., Potdar, M. \& Mahadik, K.R. 2009. Application of Quality Control Principles to Herbal Drugs. International Journal of Phytomedicine 12, 4-11.

[5] Folashade, K.O., Omoregie, E.H. and Ochogu, A.P. 2012. Standardization of herbal medicines A review. International Journal of Biodiversity and Conservation 4(3), 101-112.

[6] World Health Organization 2011, Quality Control Methods for Herbal Materials, World Health Organization, Japan.

[7] Yongyu, Z., Shujun, S., Jianye, D., Wenyu, W., Huijuan, C., Jianbing, W. and Xiaojun, G. 2011. Quality Control Method for Herbal MedicineChemical Fingerprint Analysis. Quality Control of Herbal Medicines and Related Areas. InTech Open.

[8] Pawaskar, S.M. and Sasangan, K.C. 2017. Pharmacognostical and Physicochemical Evaluation of Cynodon dactylon Linn. Pers. Leaf Extract. International Journal of Pharmaceutical and Sciences Research 8(9), 3855-3862.

[9] Yesuf, A., Wondimeneh, Y., Gebrecherkos, T. and Moges, F. 2016. Occurrence of Potential Bacteria Pathogens and their Antimicrobial Susceptibility Patterns Isolated from Herbal Medicinal Products Sold in Different Markets of Gondar Town, Northwest Ethiopia. International Journal of Bacteriology 1-11.

[10] Lead Poisoning and Health. 2017. World Health Organization (WHO). Accessed $6^{\text {th }}$ April 2018. http://www.who.int/mediacentre/factsheets/fs $379 /$ en/.

[11] Mercury and Health. 2017. World Health Organization (WHO). Accessed $6^{\text {th }}$ April 2018. http://www.who.int/mediacentre/factsheets/fs361/ en/.

[12] Arsenic. 2017. World Health Organization (WHO). Accessed $6^{\text {th }} \quad$ April 2018. http://www.who.int/mediacentre/factsheets/fs372/ en/.

[13] Kunle, O.F., Omoregie, E.H. and Ochogu, A.P. 2012. Standardization of Herbal Medicines - A Review. International Journal of Biodiversity and Conservation 4, 101-112.

[14] Spink, J. and Moyer, D.C. 2011. Defining the Public Health Threat of Food Fraud. Journal of Food Science 76(9), 157-163.

[15] Masand, S., Madan, A. and Balian, S.K. 2014. Modern Concept of Storage and Packaging of Raw Herbs in Ayurveda. International Journal of Research in Ayurveda and Pharmacy 5, 242-245.

[16] World Wide Fund, The importance of Forests Cannot be Underestimated. Accessed $2^{\text {nd }}$ April 2018.

http://wwf.panda.org/about_our_earth/deforestati on/importance forests/.

[17] WHO Guidelines on Good Manufacturing Practices (GMP) for Herbal Medicines. 2007. World Health Organization (WHO). Geneva: World Health Organization. Accessed $6^{\text {th }}$ April 2018. http://www.who.int/iris/handle/10665/43672.

[18] Choudhary, N. and Sekhon, B.S. 2011. An Overview of Advances in the Standardization of Herbal Drugs. Indian Journal of Pharmaceutical Education and Research 2(2), 55-70.

[19] Kumari, R. and Kotecha, M. 2016. A Review on the Standardization of Herbal Medicines. International Journal of Pharma Sciences and Research 7(2), 97-106.

[20] Shulammithi, R., Sharanya, M., Tejaswini, R. and Kiranmai, M. 2016. Standardization and quality evaluation of herbal drugs. Journal of Pharmacy and Biological Sciences 11(5), 89-100.

[21] International Organization of Standardization, ISO 9000 Family-Quality Management. Accessed $10^{\text {th }}$ April 2018. https://www.iso.org/iso-9001-qualitymanagement.html.

[22] Frost, R. 2004. International Organization for Standardization. Quality Assurance Journal 8, 198-206.

[23] Peter, K.V. 2006. Handbook of Herbs and Spices, Woodhead Publishing Limited, USA.

[24] Motarjemi, Y. 2016. Food Safety Management: Handbook of Hygiene Control in the Food Industry, $2^{\text {nd }} E d$. Woodhead Publishing Series.

[25] Sikora, T. and Strada, A. 2006. Safety and Quality Assurance and Management Systems in Food Industry: An overview.

[26] Pandey, A.k. and Savita. 2017. Harvestingand post-harvest precessingof medicinal plants: 
problems and prospects. The Pharma Innovation Journal 6(12), 229-235.

[27] World Health Organization 2003, WHO Guidelines on Good Agricultural and Collection Practices (GACP) for Medicinal Plants, World Health Organization, Luxembourg.

[28] Moreira, D.L., Teixerira, S.S., Monteiro, M.H.D., Oliveira, A.C.A.X. and Paumgartten, F.J.R. 2014. Traditional use and safety of herbal medicines. Brazilian Journal of Pharmacognosy 24, 248-257.
[29] Barnes, J, Anderson, LA \& Phillipson, JD 2007, Herbal Medicines, $3^{\text {rd }}$ ed, Pharmaceutical Press, UK.

[30] Shirwaikar, A., Verma, R., Lobo, R. and Shirwaikar, A. 2009. Phytotheraphy-Safety aspects. Natural Products Radiance 8(1), 55-63.

[31] Balachandran, P., Wei, F., Lin, R.C., Khan, I.A. and Pasco, D.S. 2005. Structy Activity Relationship of aristolochic acid analogues: Toxicity in cultured renal epithelia. Official Journal of the International Society of Nephrology 67(5), 1797-1805. 